\title{
Performance Modeling of Location Management Using Multicasting HLR with Forward Pointer in Mobile Networks
}

\author{
Dong Chun Lee ${ }^{1}$ Sung-Kook $\mathrm{Han}^{2}$, and Young Song Mun ${ }^{3}$ \\ ${ }^{1}$ Dept. of Computer Science Howon Univ., Korea \\ e-mail:ldch@sunny.howon.ac.kr \\ ${ }^{2}$ Dept. of Computer Eng. Wonkwang Univ., Korea \\ ${ }^{3}$ Dept. of Computer Science Soongsil Univ., Korea
}

\begin{abstract}
We propose a new location management called Multicasting HLR with Forward Pointer (MHFP) which exploits receiver side call locality in Mobile Networks (MN). When a call is established, Multicasting HLR (MH) records the caller's VLR ID according to the callee. Periodically, MH ranks the VLRs and determines which VLRs frequently make calls to the callee. During a location registration process, $\mathrm{MH}$ sends the terminal's location information to the determined VLRs. And also, when a mobile terminal frequently moves between two Registration Areas (RAs) or within a small area, the terminal's location information does not register to HLR but to VLR using the Forwarding Pointer (FP) with unit length and link both sides.
\end{abstract}

\section{Introduction}

For mobility management scheme in the MN, the standard commonly used in North America is the EIA / TIA Interim Standard 41 (IS-41), and in Europe the GSM[2]. And whenever a terminal crosses a RA or a call originates, the HLR should be updated or queried. Frequent DB accesses and message transfers may be cause the HLR bottleneck problem and then degrade the system performance. A number of related works have been reported to reduce overhead traffic of the HLR. In [7], [8], a Location Forwarding Strategy is proposed to reduce the signaling costs for location registration. A Local Anchoring Scheme is introduced in [1], [4]. Under these schemes, signaling traffic due to location registration is reduced by eliminating the need to report location changes to the HLR. Hierarchical database system architecture is introduced in [3]. These schemes can reduce both signaling traffics due to location registration and call tracking using the properties of call locality and local mobility. We propose a new location management scheme to reduce the location overhead traffic of HLR.

\section{IS-41 Standard Scheme}

The whole MN coverage area is divided into cells. Each mobile terminal within a cell communicates with the network through a Base Station (BS) which is 
installed inside the cell. These cells are grouped together to form larger areas called RAs. All BSs, belonging to a given RA, are wired to a Mobile Switching Center (MSC). In this paper, we assume that the VLR is co-located with the MSC and a single HLR in the network [4]. In order to locate a terminal effectively when a call arrives, each terminal is required to report its location whenever it enters a new RA. We call this reporting process location registration. In order to track a call to the proper terminal, the HLR and the VLRs are queried to find the current RA, and all cells within the RA are paged to find it. Within the call tracking, we call the queries to the HLR and the VLRs as the Search. According to the IS-41 location strategy, the HLR always knows exactly the ID of the serving VLR of a mobile terminal. We outline the major steps of the IS-41 location registration. (For the details, refer to [4], [9].)

1. The mobile terminal sends a Registration Request (REGREQ) message to the new VLR.

2. The new VLR checks whether the terminal is already registered. If not, it sends a Registration Notification (REGNOT) message to the HLR.

3. The HLR sends a Registration Cancellation (REGCANC) message to the old VLR. The old VLR deletes the information of the terminal.

and the IS-41 call tracking is outlined as follows:

1. The VLR of caller is queried for the information of callee. If the callee is registered to the VLR, the SEARCH process is over and the call is established. If not, the VLR sends a Location Request (LOCREQ) message to the HLR.

2. The HLR finds out to which VLR the callee is registered, and sends a Routing Request (ROUTREQ) message to the VLR serving the callee. The VLR finds out the location information of the callee.

3. The serving MSC assigns a Temporary Local Directory Numbers (TLDN) and returns the digits to the VLR which sends it to the HLR.

4. The HLR sends the TLDN to the MSC of the caller.

5. The MSC of the caller establishes a call by using the TLDN to the MSC of the callee.

Among the above 5 steps, the call tracking process is composed of step 1 and step 2.

\section{MHFP Scheme}

When a call is established, MH records the caller's VLR ID according to the callee. Periodically, MH ranks the VLRs and determine which VLRs frequently make calls to the callee. During a location registration process, MH sends the terminal's location information to the determined VLRs. And also, when a mobile terminal frequently moves between two Registration Areas (RAs) or within a small area, the terminal's location information does not register to HLR but to VLR using the Forwarding Pointer (FP) with unit length and with both side links. Fig. 1 shows one case of location registration when lenth of FP is longer 
than 1, and the VLR in the starting point of FP is not multicasting. And Fig. 2 also shows one case of call tracking when the receiving terminal has multicasting data, and the VLR has FP of the receiving terminal.

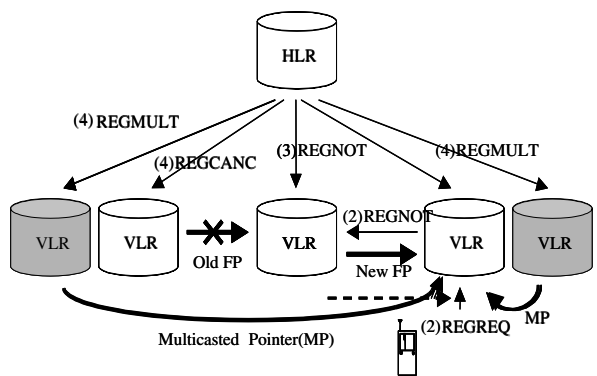

Fig. 1. Location registration $(\mathrm{m}=2)$.

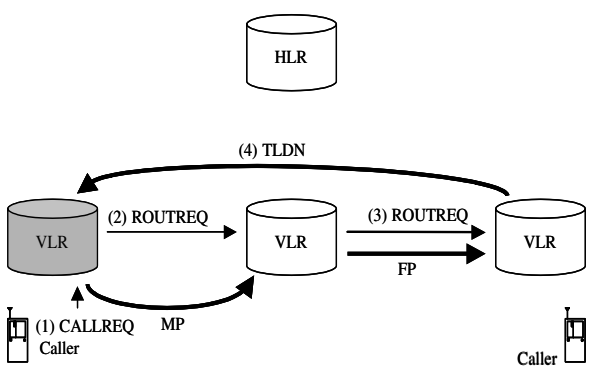

Fig. 2. Call tracking.

Here REGMULT represents the registration multicasting and $m$ represents the number of VLR to multicast the location information. We outline the major steps of location registration as follows (see Fig. 1):

- The REGREG message from a terminal is transferred to the VLR that messages a new RA. The VLR transfers the REGNOT message to previous VLR.

Previous VLR performs the query by terminal number.

- If the FP of RT exists in the end point in query result,

- The previous VLR of terminal transfers REGNOT message including FP information to the HLR. The previous VLR of terminal make new FP to current VLR of terminal. The HLR performs the query by number of received terminal.

- If length of FP is longer than 1, and the VLR in the starting point of $\mathrm{FP}$ is not multicasting ,

- The REGCANC message is transferred to the VLR which has previous FP of terminal. The REGMULT message is transferred to the multicasting VLRs.

- If length of FP is longer than 1, and the VLR in the starting point of $\mathrm{FP}$ is multicasting,

- The REGMULT message is transferred to the multicasting VLRs.

- Else if length of FP is less than 1,

- Previous VLR of terminal makes new FP to current VLR of terminal.

And call tracking is outline as follows (see Fig.3):

- The sending terminal (ST) requests a call to the VLR. The VLR performs the query by number of RT.

- If the RT exists in query result,

- The TLDN is assigned to the RT via the MSC.

- Else if the FP of RT exists in query result,

- The ROUTREQ message is transferred to the VLR directed by the FP. The VLR directed by the FP assigns TLDN via the MSC and transfers to the VLR of ST. 
- Else if the multicasting information of the RT exists in query result,

- The ROUTREQ message is transferred to the VLR directed by multicasting information. The VLR directed by multicasting information performs the query by number of RT.

- If RT has multicasting information and the VLR has in the RT in query result,

- The TLDN is assigned to the RT via the MSC and is transferred to the VLR of sending terminal.

- Else the RT has multicasting information, and the VLR has the FP of RT,

- The ROUTREQ message is transferred to the VLR directed by the FP. The VLR directed by the FP assigns TLDN via the MSC and transfers to the VLR of ST.

- Else if the multicasting information of the RT doesn't exists in query result,

- The VLR of ST transfers LOCREQ of message to the HLR. The HLR find the VLR of RT by query and transfers to ROUTREQ. The VLR which receive ROUTREQ message performs the query by number of RT.

- If the RT must search to the HLR and the VLR directed by the HLR has the RT,

- The TLDN is assigned to the RT via the MSC and is transferred to the HLR. The HLR transfer the TLDN to the VLR of ST.

- Else the RT must search to the HLR and VLR directed by HLR has the FP of RT,

- The ROUTREQ message is transferred to the VLR directed by the FP. The VLR directed by the FP assigns TLDN via the MSC and transfers to the VLR of ST. The HLR transfer The TLDN to the VLR of ST.

In selection of multicasting objects, m, Fig. 3 shows the conceptual structure of the CL field and RL field.

\begin{tabular}{|l|l|l|l|l}
\hline VLR ID 1 & Counter & VLR ID 2 & Counter & $\bullet$ \\
\hline
\end{tabular}

(a) Caller list field.

\begin{tabular}{|l|l|l|l}
\hline VLR ID 1 & VLR ID 2 & VLR ID 3 & $\bullet$ \\
\hline
\end{tabular}

(b) Rank list field.

Fig. 3. Conceptual structure of the CL field and RL field.

Fig. 3 (a), the CL field consists of the pairs of the ID of frequently calling VLR and number of calls from it. Every established call adds a new pair or increases the number of existing calls. The CL fields are distributed over the HLR and multicast VLR recording the established calls, and merged into HLR periodically to construct the RL field. In Fig.3 (b), each element of RL field is an ID of VLR and the ID of VLR calling more frequently course before. The RL field exists only in the user profile of HLR while the CL field is dispersed over VLR and HLR. 


\section{Analytical Model}

Form the user's point of view, the end-to-end service delay (location registration or call tracking) will be an important performance metric. To evaluate this end-to-end delay, we treat the database of MN as Jackson's network. The service time of each database operation is assumed to be a major delay, and we do not consider a link cost [5], [6]. We assume that there are $n$ VLRs and one HLR in the system. The HLR is assumed to have an infinite buffer and single exponential server with the average service time $\frac{1}{\mu_{h}}$. Likewise, the VLR is assumed to have an infinite buffer and single exponential server with the average service time $\frac{1}{\mu_{v}}$. We assume that within a RA, the location registration occurs in a Poisson process with rate $\lambda_{u}$ and the call origination occurs in a Poisson process with rate $\lambda_{c}$. With these assumptions, the MN using IS-41standard as a mobility management method becomes Jackson's network [10] as shown in Fig.4.

$\lambda_{l r}$ and $\lambda_{t t}$ represent the average arrival rate of REGCANC message and the average arrival rate of ROUTREQ message, respectively. $\lambda_{h}$ represents the average arrival rate of messages to the HLR from other VLRs, and by the Burke's theorem [10] it is the same as the average departure rate of messages from the HLR. $\mathrm{P}_{v o}$ is the probability the departure message from the VLR leaves the system. $\mathrm{P}_{v h}$ is the probability the departure message from the VR enters the HLR. $\mathrm{P}_{h v}$ is the probability the departure message from the HLR enters one of $n$ VLRs. From the definition of $\lambda_{l r}$ and $\lambda_{t t}$, we know that these messages get out of the system after going through the VLR.

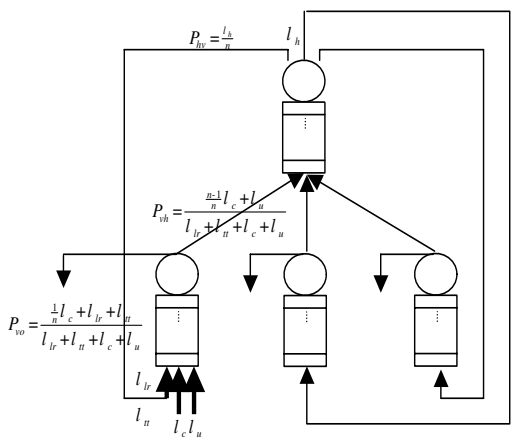

Fig. 4. Jackson's network modeling of the IS-41standard scheme.

$\lambda_{u}$ enters the VLR in the form of REGREQ message, and after receiving services from the VLR it is delivered to the HLR in the form of REGNOT message. After receiving services from the VLR, $\frac{1}{n} \lambda_{c}$ get out of the system because the probability the callee is in the same RA with the caller is $\frac{1}{n}$. So, we have

$\mathrm{P}_{v o}=\frac{\frac{1}{n} \lambda_{c}+\lambda_{l r}+\lambda_{t t}}{\lambda_{l r}+\lambda_{t t}+\lambda_{c}+\lambda_{t t}}$ 
$\mathrm{P}_{v h}=\frac{\frac{n-1}{n} \lambda_{c}+\lambda_{t t}}{\lambda_{l r}+\lambda_{t t}+\lambda_{c}+\lambda_{t t}}$

$\mathrm{P}_{h v}=\frac{\lambda_{h}}{n}$.

By the property of Jackson's network, we have

$\lambda_{h}=n \times\left(\frac{n-1}{n} \lambda_{c}+\lambda_{t t}\right)$.

From $\frac{\lambda_{h}}{n}=\lambda_{l r}+\lambda_{t t}$, we have

$\lambda_{l r}=\lambda_{t t}$,

$\lambda_{t t}=\frac{n_{1}}{n} \lambda_{c}$

Though Eqs. (5) and (6) could be directly inferred from the definitions of $\lambda_{c}$ and $\lambda_{u}$, we lead these equations through Eqs. (1)-(4) to help understand how $\lambda_{c}$ and $\lambda_{u}$ are delivered to HLR and VLRs and how many messages arrive at these DBs on average using the property of Jackson's network. We will repeat these steps in the following Jackson's network modeling the proposed scheme.

Now, let $W_{v}$ and $W_{h}$ represent the average system time (queue plus service) in the VLR and the average system time in the HLR, respectively. By the Little's Rule [10], $W_{v}$ and $W_{h}$ becomes

$W_{v}=\frac{1}{\mu_{v}-\left(\lambda_{l r}+\lambda_{t t}+\lambda_{c}+\lambda_{t t}\right)}=\frac{1}{\mu_{v}-\left(\frac{2 n-1}{n} \lambda_{c}+\lambda_{t t}\right)}$,

$W_{h}=\mu_{h}-n\left(\frac{n-1}{n} \lambda_{c}+\lambda_{t t}\right)$.

Likewise, Fig.6 shows Jackson's network model of MHFP scheme. The focus of message flow aims at the quantity.

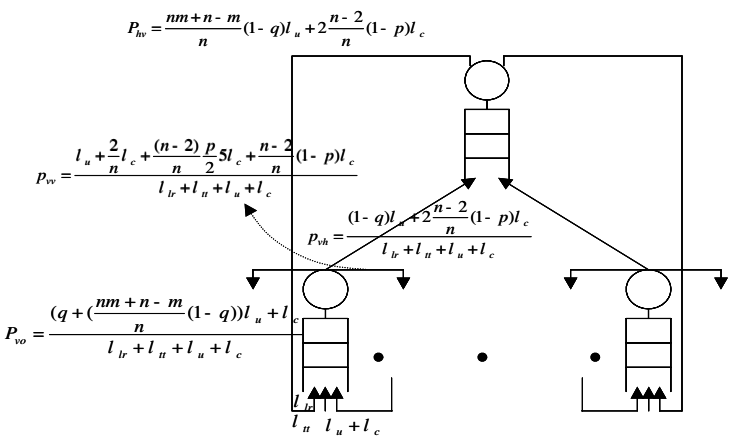

Fig. 5. Jackson's network modeling of the MHFP scheme. 
The wide difference between Fig.4 and Fig. 5 is that the number of message out coming HLR. When call managed in HLR, passing one more device dealing with multicasting can solve this problem. Another difference is what communicates call between VLR passing HLR. It is because of obtaining location information of the receiving terminal by multicasting information, $\mathrm{m}$. We need to calculate the probability that the departure message from the VLR is delivered to another VLR. We denote this probability by $P_{v v}$. Considering the definition of $\lambda_{l r}, \lambda_{t t}, \lambda_{c}, \lambda_{u}$ as follows:

$P_{v o}=\frac{\left(q+\frac{n m+n-m}{n}(1-q)\right) \lambda_{u}+\lambda_{c}}{\lambda_{u}+\lambda_{l r}+\lambda_{c}+\lambda_{t t}}$.

$P_{v h}=\frac{(1-q) \lambda_{u}+2 \frac{n-1}{n}(1-p) \lambda_{c}}{\lambda_{u}+\lambda_{l r}+\lambda_{c}+\lambda_{t t}}$.

$P_{v v}=\frac{\lambda_{u}+\frac{2}{n} \lambda_{c}+\frac{(n-2)}{n} \frac{p}{2} 5 \lambda_{c}+\frac{n-2}{n}(1-p) \lambda_{c}}{\lambda_{u}+\lambda_{l r}+\lambda_{c}+\lambda_{t t}}$.

$P_{h v}=\frac{n m+n-m}{n}(1-q) \lambda_{u}+2 \frac{n-1}{n}(1-p) \lambda_{c}$.

$\mathrm{P}$ represents probability that multicasting message search to VLR of receiving terminal, and $\mathrm{q}$ is probability that the FP has less than 1. By the definition of $\lambda_{t t}$ and $\lambda_{l r}$, we obtain

$\lambda_{t t}=2 \frac{n-1}{n}(1-p) \lambda_{c}$,

$\lambda_{l r}=\frac{n m+n-m}{n}(1-q) \lambda_{u}$.

From the property of Jackson's network, we have

$\lambda_{h}=n\left(2 \frac{n-1}{n}(1-p) \lambda_{c}+\frac{n m+n-m}{n}(1-q) \lambda_{u}\right)$.

Let $W_{v}^{\prime}$ and $W_{h}^{\prime}$ denote the average system time in the VLR and in the HLR, respectively, From the Little's Rule, $W_{v}^{\prime}$ and $W_{h}^{\prime}$ are:

$W_{v}^{\prime}=\frac{1}{\mu_{v}-\left(\lambda_{t t}+\lambda_{l n}+\lambda_{c}+\lambda_{u}\right)}$
$=\frac{1}{\mu_{v}-\left(2 \frac{n-1}{n}(1-p) \lambda_{c}+\frac{n m+n-m}{n}(1-q) \lambda_{u}+\lambda_{c}+\lambda_{u}\right)}$.

$W_{h}^{\prime}=\frac{1}{\mu_{v}-\left(2 \frac{n-1}{n}(1-p) \lambda_{c}+\frac{n m+n-m}{n}(1-q) \lambda_{u}\right)}$.

Table 1 shows the average number of arrival messages to the VLR, and the average system time in the VLR when the proposed scheme is used as 
Table 1. VLR comparisons between the MHFP and IS-41scheme

\begin{tabular}{|c|c|c|}
\hline & $\begin{array}{c}\text { Average number of arrival } \\
\text { messages }\end{array}$ & Average system time in VLR \\
\hline IS-41 & $\frac{2 n-1}{n} \lambda_{c}+2 \lambda_{u}$ & $\frac{1}{\mu_{v}-\left(\frac{2 n-1}{n} \lambda_{c}+2 \lambda_{u}\right)}$ \\
\hline MHFP & $\left(2 \frac{n-1}{n}(1-p) \lambda_{c}\right.$ & $\frac{n}{\mu_{v}-\left(2 \frac{n-1}{n}(1-p) \lambda_{c}+\frac{n m+n-m}{n}(1-q) \lambda_{u}+\lambda_{c}+\lambda_{u}\right)}$ \\
& $\left.+\frac{n m+n-m}{n}(1-q) \lambda_{u}+\lambda_{c}+\lambda_{u}\right)$ & \\
\hline
\end{tabular}

Table 2. HLR comparisons between the MHFP and IS-41scheme

\begin{tabular}{|c|c|c|}
\hline & $\begin{array}{c}\text { Average number of arrival } \\
\text { messages }\end{array}$ & Average system time in VLR \\
\hline IS-41 & $n\left(\frac{n-1}{n} \lambda_{c}+\lambda_{u}\right)$ & $\frac{1}{\mu_{h}-n\left(\frac{n-1}{n} \lambda_{c}+\lambda_{u}\right)}$ \\
\hline MHFP & $\begin{array}{c}n\left(2 \frac{n-1}{n}(1-p) \lambda_{c}\right. \\
\left.+\frac{n m+n-m}{n}(1-q)\right)\end{array}$ & $\left.\frac{n+n+n-m}{n}(1-q) \lambda_{u}\right)$ \\
\hline
\end{tabular}

compared to those of IS-41scheme. And table 2 shows the average number of arrival messages to the HLR, and the average system time in the HLR. As shown in table 1 and table 2, the proposed scheme distributes messages from the HLR to the VLRs, and it also reduces the average system time in the HLR with the small increase of the average system time in the VLRs. Based on the delay times of the HLR and the VLR, we can calculate the mobility management costs for IS-41 standard and the proposed scheme as follows.

- The IS-41scheme for mobility management cost.

(1) $W_{I S-41 L}\left(\right.$ Location registration cost) $=W_{v}+W_{h}+W_{v}$.

(2) $W_{I S-41 C}($ Call tracking cost $)=\frac{1}{n} W_{v}+\frac{n-1}{n}\left(W_{v}+W_{h}+W_{v}\right)$.

(3) $W_{I S-41 M}$ (Mobility management cost)

$$
=\frac{\lambda_{u}}{\lambda_{c}+\lambda_{u}} \times \mathrm{W}_{I S-41 L}+\frac{\lambda_{c}}{\lambda_{c}+\lambda_{u}} \times \mathrm{W}_{I S-41 C} .
$$

- The proposed scheme for mobility management cost.

(1) $W_{\text {Proposed } L}^{\prime}($ Location registration cost)

(2) $W_{\text {ProposedC }}^{\prime}($ Call tracking cost)

$$
=q \times 2 W_{v}^{\prime}+(1-q)\left(2 W_{v}^{\prime}+W^{\prime} h\right) .
$$

$=\left(\frac{1}{n}\right) \times 4 W_{v}^{\prime}+\frac{n-2}{n} \times \frac{p}{2} \times 7 W_{v}^{\prime}+\frac{n-2}{n}$
(3) $W_{\text {Proposed }}^{\prime}($ Mobility management cost $)$

$$
=\frac{\lambda_{u}}{\lambda_{c}+\lambda_{u}} \times W_{\text {Proposed } L}^{\prime}+\frac{\lambda_{c}}{\lambda_{c}+\lambda_{u}} \times W_{\text {Proposed } C}^{\prime} .
$$

\section{Numerical Results}

To get numerical results, we use the same value of system parameters as those in [2], $\mathrm{n}=128$, for example. From these parameters, the average occurrence rate of location registration in an RA, $\lambda_{u}$, is calculated as $\lambda_{u}=$ 
$5.85 / \mathrm{s}$. And the average call origination rate in an $\mathrm{RA}, \lambda_{c}$, is calculated as $\lambda_{c}=8.70 / \mathrm{s}$. We assume that the average service rates of HLR and VLR are $\mu_{h}=2000 / \mathrm{s}, \mu_{v}=1000 / \mathrm{s}$, and Probability $\mathrm{p}$ is 0.5 .

In figures, X-coordinate shows probability $\mathrm{q}$ that the $\mathrm{FP}$ has less than 1 and Y-coordinate shows the ratio dividing the result value in IS-41scheme into the result value in proposed scheme. If the ratio value is 1 , the IS-41scheme performance is equal to proposed scheme performance. If it has greater than 1 , the performance of proposed scheme is superior to the performance of IS-41scheme. And if it has less than 1, the performance of IS-41scheme is superior to the performance of proposed scheme. The example of the lower graph can show $\mathrm{m}$ of multicasting factor. It is determined whether we multicast the location information of terminal into several VLRs.

In Fig.6, we can know that the results of performance are superior to IS41scheme. As multicasting the location registration, this result becomes a matter of course. If $\mathrm{m}$ increases gradually, the level of performance is lower. In Fig.7, regardless of $\mathrm{m}$, the graph is determined by the probability q, but $\mathrm{m}$ has no effect upon the value. Otherwise, a relation of between $\mathrm{m}$ and probability $q$ is actually represented by an expression but also, it is facts that $\mathrm{m}$ has no effect upon the value. When $\mathrm{m}$ is 3, we may assume that probability $\mathrm{q}$ is 0.5 . If probability $\mathrm{q}$ is 0 , the number of the call tracking message is equal to the number of the location registration message.

Fig. 8 shows message ratio in mobility management cost. If probability $q$ is 0.5 , and $\mathrm{m}$ is 3 , the value is equal to 0.87 in short. It is fact that proposed scheme is 1.15 times as many messages as IS-41scheme.

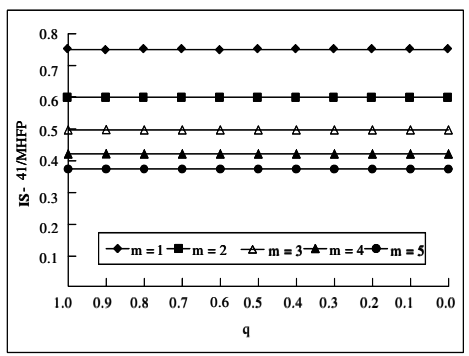

Fig. 6. Message number ratio in registration cost.

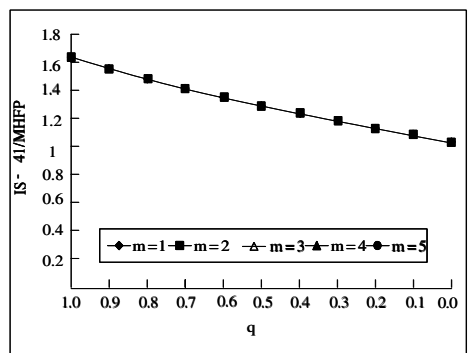

Fig. 7. Message number ratio in call tracking cost.

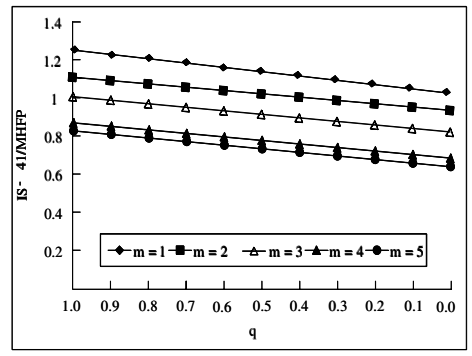

Fig. 8. Message number ratio in mobility management cost. 


\section{Conclusions}

We propose MHFP scheme to reduce the location traffic of HLR. In numerical results, the proposed scheme has lower value in the number of call tracking message and the management delay time of HLR than the IS-41scheme. Especially, the management delay time of HLR has more performance than the IS-41scheme. The proposed scheme can be expected to have the prominent performance advancing for mobile management in the $3 \mathrm{G}$ mobile networks.

\section{Acknowledgments}

This work is supported in part by the ministry information Communication Communication of Korea, under the "Support Project of University information Technology Research Center(ITRC)" supervised by KIPA".

\section{References}

1. I. F. Akyildiz, J, McNair, J, Ho, H. Uzunalioglu, W. Wang, "Mobility Management in Current and Future Communication Networks," IEEE Network, Jul./Aug., 1998, pp. 39-49

2. R. Jain, Y. B. Lin, C. N. Lo., and S. Mohan, "A Caching Strategy to Reduce Network Impacts of PCS," IEEE Jour. on Selected Areas in Com., Vol. 12, No. 8, 1994, pp. 1434-1445

3. C. L. I, G. P. Plooini and R. D. Gitlin, "PCS Mobility Management using the Reverse Virtual All Setup Algorithm," IEEE/ACM Trans. Vehicle. Tech, 1994, pp. 1006-1010

4. T. Russel, Signaling System $\sharp 7$, McGraw-Hill, 1995

5. J. Z. Wang, "A Fully Distributed Location Registration Strategy for Universal Personal Communication Systems," IEEE Personal Com., First Quarter 1994, pp. 42-50995.

6. C. Eynard, M. Lenti, A. LOmbardo, O. Marengo, S. Palazzo, "Performance of Data Querying Operations in Universal Mobile Telecommunication System (UMTS)."Proceeding of IEEE INFOCOM '95, Apr. 1995, pp. 473-480

7. T. H. Cormen, C. E. Leiserson, R. L. Rivest, Introduction to Algorithms, MIT Press and McGraw-Hill, 1990

8. N. Shivakumar, J. Widom, "User Profile Replication for Faster Location Lookup in Mobile Environment," Proceeding of ACM MOBICOM, Nov. 1995, pp. 161-169

9. Y. -B. Lin, "Reducing Location Update Cost in a PCS Network," IEEE/ACM Trans. Networking Vol. 5, Feb. 1997, pp. 25-33

10. L. Kleinrock, " Queuing Systems", Vol. 1, Wiley-Interscience, 1975 